\title{
Transcatheter approaches for mitral valve regurgitation
}

\author{
Juri Sromicki, Luca Vicentini, Mizuki Miura, Alberto Pozzoli, Mathias Van Hemelrijck, Maurizio Taramasso, \\ Francesco Maisano
}

Department of Cardiac Surgery, Universitäts Spital Zürich (USZ), Heart Center University Hospital of Zurich, Zürich, Switzerland

Contributions: (I) Conception and design: J Sromicki, M Taramasso, F Maisano; (II) Administrative support: J Sromicki, L Vicentini, M Taramasso,

F Maisano; (III) Provision of study materials or patients: J Sromicki, L Vicentini, M Taramasso, F Maisano; (IV) Collection and assembly of data: J Sromicki, M Miura, A Pozzoli, M Van Hemelrijck; (V) Data analysis and interpretation: J Sromicki, L Vicentini, M Miura, M Taramasso; (VI) Manuscript writing: All authors; (VII) Final approval of manuscript: All authors.

Correspondence to: Juri Sromicki. Department of Cardiac Surgery, Universitäts Spital Zürich, Heart Center University Hospital of Zurich, Zurich, Switzerland. Email: juri.sromicki@usz.ch.

\begin{abstract}
Surgical repair for a longtime was and currently is regarded as the gold-standard for the treatment of mitral regurgitation (MR). With an aging population, significant MR became not only more prevalent, but also fundamental for development of transcatheter devices for patients at high surgical risk. After successful attempts in transcatheter mitral valve reconstruction two decades ago, successful transcatheter mitral valve replacement (TMVR) was performed for the first time in 2012. Currently several repair-systems are available and a couple of them proved to be even superior to conventional surgery in selected patient populations. Since the mitral valve (MV) apparatus is a complex anatomical structure, progress in development of TMVR took longer than in transaortic valve replacement (TAVR). We aim at giving insight in current standards of transcatheter treatments for severe MR by critically reviewing the latest literature.
\end{abstract}

Keywords: Transcatheter; mitral regurgitation (MR); functional mitral valve regurgitation (FMR); degenerative mitral valve regurgitation (DMR); transcatheter mitral valve replacement (TMVR)

Received: 19 August 2019; Accepted: 05 September 2019; Published: 30 September 2019.

doi: 10.21037 /jovs.2019.09.05

View this article at: http://dx.doi.org/10.21037/jovs.2019.09.05

\section{Introduction}

Mitral regurgitation is the most common heart-valve disease and associated with poor patient outcome (1). Increasing prevalence of mitral regurgitation (MR) in the elderly, longer life-expectancy and the fact that about half of the patients with mitral regurgitation are treated with medical therapy alone, have contributed to the fact, that treatment-strategies for MR lately have come into focus of both surgeons and interventional cardiologists likewise. Untreated MR results in chronic volume overload of the left ventricle $(\mathrm{LV})$ and is associated with high morbidity and mortality. Cases of severe MR with decreasing LVEF need to be addressed early and medical and interventional treatment-options should be evaluated carefully (2).

While for a longtime alongside medical therapy surgery was the only accessible treatment for MR, elder patientpopulation and associated comorbidities prohibitive for operations on heart-lung-bypass, have led to the development of transcatheter-approaches for patients at high surgical risk.

If performed in experienced centers, surgical mitral valve (MV)-repair still reveals excellent results and despite transcatheter-developments it is still gold-standard in treatment of degenerative MR in symptomatic patients at considerable risk for open-heart-surgery (3).

While development of transaortic valve replacement (TAVR) quickly led to a paradigm-shift in treatment of severe aortic stenosis, transcatheter-techniques in MR not only took longer in their development, but still are lacking of equally wide acceptance as TAVR. 


\section{Classification and current recommendations}

Since the MV apparatus is more complex than the aortic root, classification of MR by both etiology and severity is more demanding than in the aortic valve.

The most commonly used classification (CarpentierClassification) divides MR into three different groups, based on annular distension, normal or pathological leafletmotion (4).

The severity of MR is generally assessed by transthoracic echocardiography (TTE). More detailed insights on the anatomy and the underlying pathomechanisms of MR can be given by transesophageal echocardiography (TEE). Especially in degenerative MR, 3D-TEE has proven to be a valuable tool to localize and describe leaflet-prolapse or flail. CT-scan can give additional information on anatomy, proximity of the circumflex artery to the MV-annulus and tissue-calcifications.

The European (5) and American (6) guidelines both state, that MV-repair should be the treatment of choice whenever feasible. In patients with depressed LV-function and symptoms despite extended heart failure (HF)-medication, percutaneous edge-to-edge-repair, may be considered after discussion in a Heart Team.

Different surgical and interventional techniques were developed in the last years, lots of them still seeking for market approval. While transcatheter edge-to-edge-repair is widely accepted, devices addressing other parts of the valve are still rather seldomly used. Recent published evidence on the use of the MitraClip-device in patients with severe secondary MR and depressed LV-Function, is expected to widen the indication for transcatheter edge-to-edge-repair in the next revision of the valvular guidelines.

\section{Transcatheter MV-repair}

\section{Leaflet-repair edge-to-edge}

\section{MitraClip}

Used in over 70,000 patients worldwide, MitraClip is by far the most significant transcatheter MV-repair-device currently in use. Encouraged by the experiences of edge-toedge-repair in open-heart-surgery by Otavio Alfieri (7), the MitraClip-device has been developed and first implanted in a human being in 2003. Demonstrating a positive safety profile, significant reduction in MR and improvement in patient symptoms, CE-Mark [2008] and FDA-approval for treatment of degenerative MR in non-surgical candidates [2013] were obtained (8).
Guided by TEE and fluoroscopy the device is inserted through the femoral vein and introduced to the left atrium by atrial-transseptal puncture. Pushed through the MV, the free edge of the anterior and posterior leaflet can be grasped at the site of malcoaptation and clipped together (Figures 1,2). Compared to the widely used NTR-Clip, the newer generation XTR-Clip has longer arms, allowing easier grasping of the leaflets. Proper Clip-selection may be crucial to prevent MV pressure gradient (10). The fourthgeneration MitraClip-device, MitraClip G4, has received U.S. Food and Drug Administration (FDA) approval in July 2019. It comes in four different sizes, enables the operators to control gripper-arms independently and allows monitoring of left atrium pressure by a pressure-line incorporated in the delivery-system.

Randomized evidence for the use of MitraClip in MR was obtained mainly by the EVEREST-TrialSeries. While EVEREST-I in 27 patients proved safety and effectiveness of the procedure (11), the EVEREST II-Study in 279 patients with moderate to severe MR, compared treatment-results of MitraClip to surgical MVrepair and showed that the composite endpoint of freedom from death, surgery or $M R \geq 3+$ at 5 -year follow up was $44.2 \%$ in the percutaneous repair group, and $64.3 \%$ in the surgical group respectively $(\mathrm{P}=0.01)$. One-year data demonstrated that safety in MitraClip was superior to surgical treatment (12), however mortality-rate at 5 -year follow-up did not differ between the surgical and interventional arm (20.8\% vs. $26.8 \%, \mathrm{P}=0.36)$. Most of the patients $(78 \%)$ in the interventional arm not meeting the composite endpoint, needed surgical repair within 6 months after Clip-implantation. In a landmark-analysis, excluding events in the first 6 months after procedure, the compositeoutcome did not differ between the two treatment-groups, proving durability of a successful MitraClip-Procedure. Surgical reconstruction showed to be more effective than MitraClip, leaving $2.5 \%$ vs. $18.8 \%$ of the patients with MR $\geq 3+$ at 5-y Follow-up. Significant improvements in LVremodeling and in functional-NYHA-Class were shown in both study-arms (13).

The analysis of EVEREST-II prospective registry data of 351 patients at high surgical risk (STS-Score $>12 \%$ ), showed that immediate MR-reduction to a grade $\leq 2+$ could be achieved in $84 \%$. At 1-year follow-up MitraClip in this patient collective led to significant MR-reduction, clinical benefit $(82 \%$ NYHA $\geq$ III to $17 \%$ NYHA $\geq$ III, $\mathrm{P}<0.0001$ ) and decreased LV-dimensions (14).

The European post-approval-trial ACCESS-EU in a 

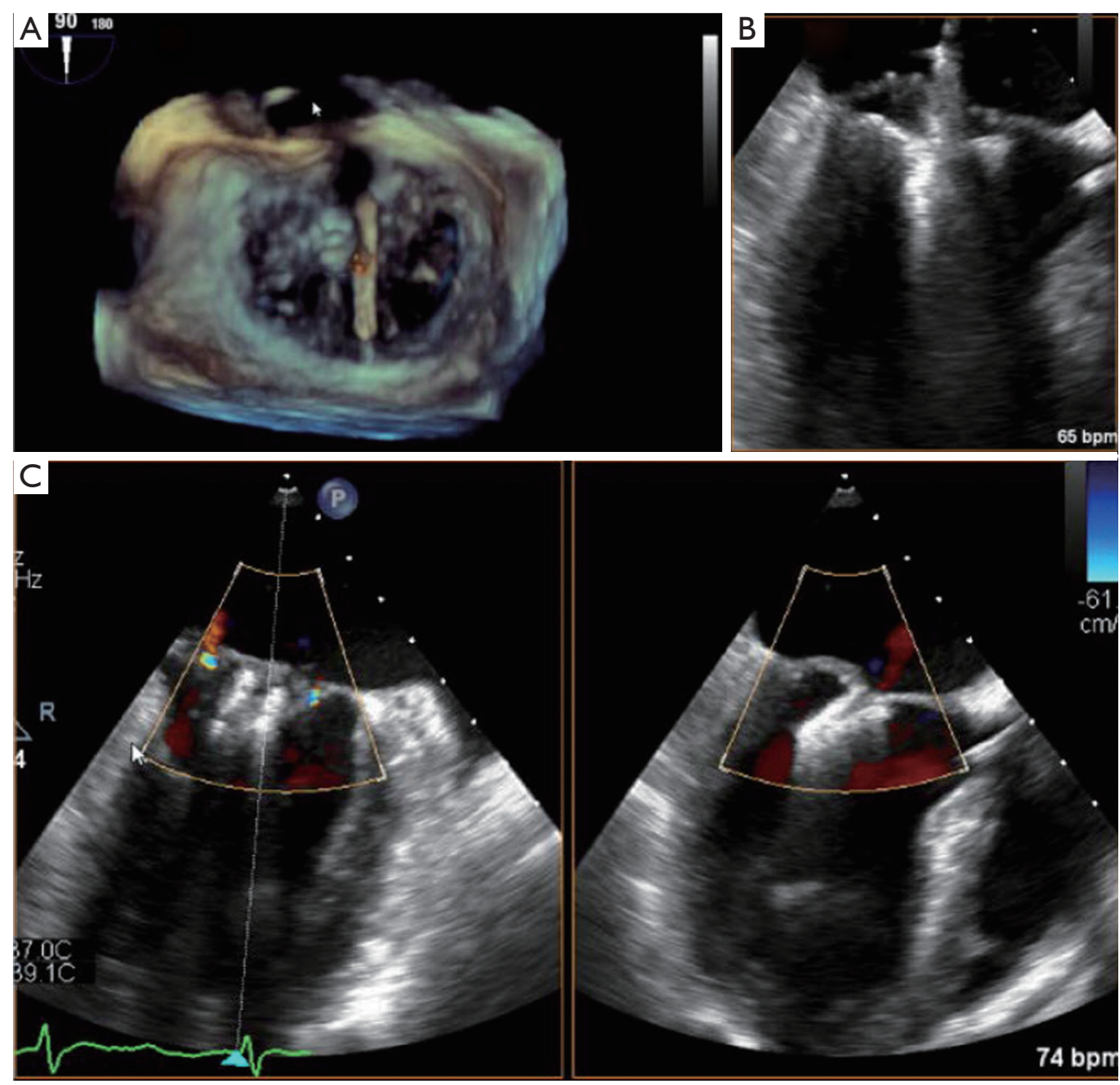

Figure 1 MitraClip-implantation. (A) Introducing the MitraClip through the valve; (B) capturing the leaflets; (C) clip in situ with trace residual MR. MR, mitral regurgitation.

real-world-population showed excellent implant-rates of $99.6 \%$ with $3.4 \%$ mortality after 30 days and only $6.3 \%$ of patients needing $\mathrm{MV}$-surgery within 12 months after MitraClip-procedure (15).

Overall improvement in MR-grade in high surgicalrisk-candidates was seen in both degenerative (DMR) and functional MR (FMR). Since FDA-approval for MitraClip was only for the treatment of DMR, researchers worldwide were eager to show the effectiveness of MitraClip in FMR too. To gain the needed data American researchers launched the COAPT (Cardiovascular Outcomes Assessment of the MitraClip Percutaneous Therapy for Heart Failure Patients with Functional Mitral Regurgitation)-trial comparing the outcomes of MitraClip to MV-surgery in severe FMR. Similar studies (MITRA-FR, MATTERHORN, RESHAPE-II) were initiated in Europe.
The respective results of COAPT $(n=610)(16)$ and MITRA-FR (n=304) (17), were presented in late 2018 and led to a controversial discussion. Both trials compared the use of the MitraClip-system on top of guidelinederived medical therapy (GDMT) to medical therapy alone. Inclusion criteria slightly differed: While patients in MITRA-FR had severe MR with a $\mathrm{RVol}>30 \mathrm{~mL}$ or EROA $>20 \mathrm{~mm}^{2}$, in COAPT the inclusion criteria were more solid including only patients with a $\mathrm{RVol}$ of $>45 \mathrm{~mL}$ or an EROA $>40 \mathrm{~mm}^{2}$. Furthermore in COAPT the patients had to remain symptomatic despite maximally-tolerated GDMT. MITRA-FR ended up being a totally negative trial showing no difference between both treatment groups in terms of survival, HF-hospitalizations or MACE at 1-year follow-up. The combined endpoint of death and HF-hospitalizations after 12 months was reached in $54.6 \%$ in the GDMT + 


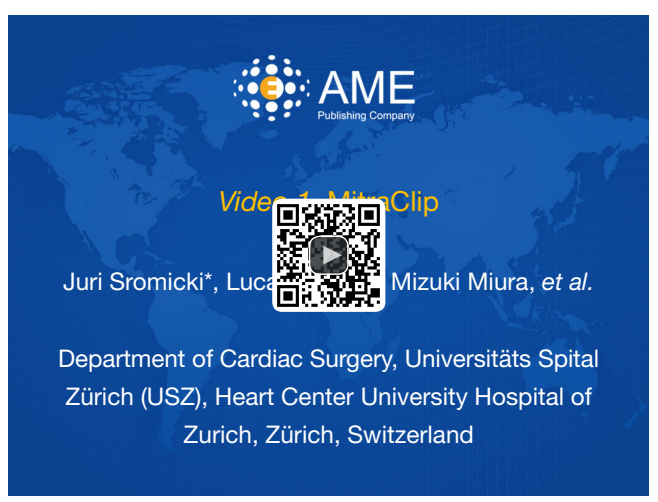

Figure 2 MitraClip (9).

Available online: http://www.asvide.com/watch/32952

MitraClip and $51.3 \%$ in the GDMT group $(\mathrm{P}=0.53)$. In the device-group, periprocedural complications were as high as $14.6 \%$. Subgroup-analyses (EROA $<30 \mathrm{~mm}^{2}, 30-40 \mathrm{~mm}^{2}$, $>40 \mathrm{~mm}^{2}$ ), showed most benefit from additional MitraClip in the group with largest EROA.

The results of COAPT showed a different outcome. Highly selected (665 patients out of 1,576 considered for enrollment), eventually 302 patients with GDMT + MitraClip were compared to 312 patients with GDMT alone. The procedures were performed in highly experienced centers only leading to a device-complicationrate of $3.4 \%$ in 1 -year follow-up. At 2 y, the use of MitraClip reduced the annualized rate of hospitalizations for $\mathrm{HF}(35.8 \%$ vs. $67.9 \%$ per patient-year, $\mathrm{P}<0.001)$ and led to a survival-benefit $(29.1 \%$ vs. $46.1 \%$ deaths from any cause, $\mathrm{P}<0.001)$. The NNT to prevent one hospitalization in $2 \mathrm{y}$ was $3.1,5.9$ to prevent one death respectively. The results were consistent in all prespecified subgroup-analyses, furthermore all secondary endpoints were in favor for MitraClip either. Hospitalization-rates were significantly lower already 30 days after MitraClip.

So why do the results differ so much? Certainly the inclusion-criteria were different. While COAPT included patients with really severe MR (EROA $>40 \mathrm{~mm}^{2}$ ) and maximally tolerated GDMT only, the enrolled patients showed only moderate $\mathrm{LV}$-dilatation with a mean left ventricular end diastolic volume (LVEDV) of $101 \mathrm{~mL} / \mathrm{m}^{2}$ compared to $135 \mathrm{~mL} / \mathrm{m}^{2}$ in MITRA-FR. Other than that, medical adjustments during the trial in MITRA-FR bring up the question, whether MITRA-FR-patients really were at best GDMT when they were enrolled. To characterize the COAPT-patients lately the term of disproportionate MR has been introduced, describing patients with very severe MR but not yet dilated LV (18).

Subgroup-analyses of the COAPT-cohort showed that MitraClip is beneficial especially in patients with MR with an EROA $>30 \mathrm{~mm}^{2}$. For EROA $<30 \mathrm{~mm}^{2}$ and already dilated LV (LVEDVI $>96 \mathrm{~mL} / \mathrm{m}^{2}$ ), representing more or less the MITRA-FR-cohort, results between GDMT with or without MitraClip did not differ.

Concluding both studies, especially in patients with disproportionate FMR, percutaneous edge-to-edge-repair improves patient-outcome and prolongs life-expectancy. As a result, on March $14^{\text {th }} 2019$ FDA approved MitraClip for treatment of selected patients with severe secondary MR who remain symptomatic despite GDMT. It is expected that the next revision of guidelines will include MitraClip for the treatment of both DMR and FMR, especially if reverse cardiac remodeling can still be achieved.

\section{PASCAL}

Due to early promising results of transcatheter edge-toedge-repair, early on the PASCAL-system was focusing on certain limitations of the MitraClip-procedure. The PASCAL-Clip has a small spacer between the arms contributing to a broader leaflet-insertion. To ease leafletcapture in the PASCAL-System both gripper-arms can be closed independently, also navigation has been improved with a delivery-system allowing direct manoeuvring in 3 planes. First-in-man experiences for the compassionate use of the PASCAL-system in 23 patients have proven feasibility with a high rate of technical success (96\%) and reduction of MR-severity ( $96 \%$ of the patients with residual MR of grade $\leq 2+$ ) (19). Recently published 30-days results of the multicenter CLASP-study showed that in 62 highly symptomatic patients with grade $M R \geq 3+$ of mixed etiology, the PASCAL-clip led to a reduction of MR to grade $\leq 2+$ in $98 \%$, grade $\leq 1+$ in $86 \%$ respectively. All-cause mortality was $1.6 \%$ and major adverse events where reported in $6.5 \%$ of the patients (20).

\section{Transcatheter annuloplasty}

\section{Cardioband}

Considering that the vast majority of patients with mitral regurgitation referred for transcatheter-treatment have FMR and encountering the high success-rates and durable results in reduction of FMR with surgical annuloplasty (21), interventional therapies mimicking direct surgical annuloplasty have been developed. Most prominent in this field, the Cardioband-device has gained CE-Mark in 
2015 for treatment of severe FMR. Using fluoroscopic and TEE-guidance, the devices' polyester sleeve is positioned at the left atrial side of the MV-annulus and sequentially anchored to the posterior site with 12-16 screws starting from the lateral, going to the medial commissure (22). Once all screws are in place, the polyester-sleeve then can be cinched under real-time TEE-control, resulting in a reduction of posterior annular dimension and consecutive improvement in leaflet coaptation (Figure 3). Preoperative CT-scan is mandatory to prevent circumflex coronary artery kinking or perforation. The ongoing ACTIVE-Trial compares the outcome of randomly assigned patients to GDMT with or without Cardioband and will give insight in short- and long-term-effects of the device. One-year-data from a European multicenter trial $(n=60)$, demonstrated a reasonable performance- and safety-profile, providing significant reduction in MR and clinical improvement. At a mean age of $72 \mathrm{y}$ at $1 \mathrm{y}$ follow-up the survival-rate was $87 \%$,

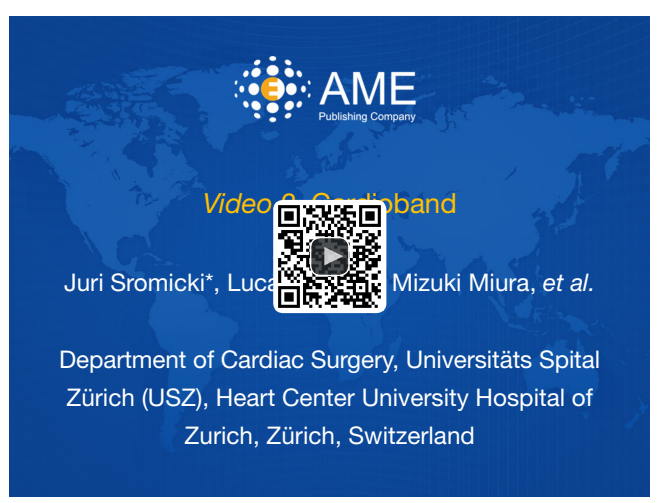

Figure 3 Cardioband (23).

Available online: http://www.asvide.com/watch/32953
$78 \%$ were free from death or a secondary intervention (24). Proper patient selection is crucial to increase technical success-rate (25). Recently presented $2 \mathrm{y}$ data of the Cardioband Mitral System CE-mark-trial showed 79\% survival at 2 years leaving $96 \%$ of these patients at $M R \leq 2+$ with sustained septolateral reduction. Not touching the MV-leaflets, Cardioband has proven to be a useful tool to regain competent leaflet coaptation. A combination with a percutaneous edge-to-edge-procedure prior to or after a Cardioband-procedure is technically feasible and has been performed many times (26) (Figure 4).

\section{Millipede IRIS}

The Millipede IRIS-transcatheter direct annuloplastysystem uses a transvenous, transseptal approach to get to the $M V$-annulus. The device itself, a semi-rigid nitinol-ring, is anchored to the $M V$-annulus with 8 screws and cinched under TEE-guidance, resulting in concentric reduction of the annular diameter. The device is repositionable and adjustable and as all other annular devices preserves the native anatomy of the MV. Initial results in a total of 7 patients (4 with on-pump deviceimplantation, 3 with transcatheter approach) have shown no adverse events, significant annular reduction $(-31.8 \%$ in septolateral diameter), good tolerance of the ring implant and reduction of MR to a grade $\leq 1+$ in all 7 patients (27). 1y-data from 11 patients presented at TCT 2018, showed the annular reduction to be constant over time. Not coated, the nitinol-ring does not require long-term Coumadintherapy.

\section{Mitralign}

Different than the other half- or full-annuloplasty-devices,
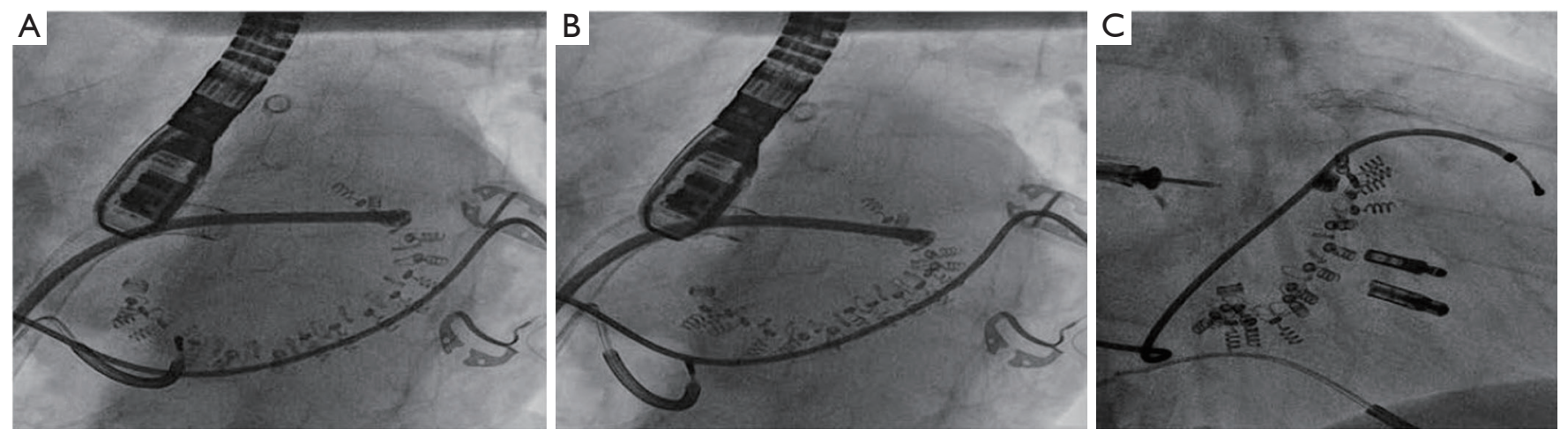

Figure 4 Combination of cardioband and MitraClip. (A) Cardioband before cinching; (B) Cardioband after cinching; (C) implantation of two additional MitraClips due to pseudoprolapse of the anterior leaflet. 
the Mitralign-System aims at annular-reduction by two single plications at both commissural sites of the MVannulus, mimicking the 1965 introduced surgical repairtechnique in functional tricuspid regurgitation by Kay (28). The device is inserted retrogradely through the aorta into the LV. TEE-guidance is used to place a double-lumen catheter at the ventricular site of the lateral and medial commissure. Two connected, pledgeted sutures are inserted at the annular base through ventriculo-atrial puncture at each commissure. Once in place, the sutures are pulled and tied leading to annular plication and reduction of the annular perimeter. To preserve valve symmetry, the procedure has to be performed at both commissures. Enrolling 70 patients in the first clinical feasibility trial, technical success was obtained in 49 patients. Limitations are commissural calcifications and annular dimensions not plicatable at two commissural sites only (29). Thirty days and 6 months safety-data showed $4.4 \%$ mortality-rate and similar stroke occurrence, furthermore $8.9 \%$ of the patients had cardiac tamponade (30). Sustained reduction of MR-severity was obtained in $50 \%$ of patients. Granted CE-mark for FMR in February 2016, the device is commercially available in Europe. In the tricuspid valve, the same concept is used in the posterior portion of the tricuspid annulus. Early clinical data suggest, that the Trialign-system tricuspid valve is safe and feasible and associated with improvement in quality-of-life (31). Future trials are needed to confirm device-success in larger cohorts and with longer follow-up.

\section{Carillon}

Indirect annuloplasty is a concept using reduction of annular dimensions by indirect annular force. The Carillon Mitral-Contour-System consists of a percutaneous indirect annuloplasty-device introduced through the right jugular vein and placed in the big cardiac vein. A proximal and a distal helical nitinol-anchor connected by a shaping arc are placed in the great cardiac vein and the coronary sinus. By cinching and shaping the vein, indirect pressure is administered on the posterior MV-annulus, leading to gentle plication of the paraannular tissue. Not touching the left side of the heart, the risk for potential stroke is maximally diminished. Gaining CE-mark-approval in 2011, more than 850 patients have been treated so far. A device modification had to be implemented due to early reports of fractures of the shaping-arc in the high-strain-region of the proximal anchor (32). Data from the TITAN-II-Trial $(\mathrm{n}=36)$ showed, that successful implantation was achieved in $83 \%$ of the patients. In 6 patients the device had to be removed due to coronary artery compromise. Clinical success was shown by sustained improvement of functional NYHAclass by 1 grade in $77.3 \%$ at 12 months (33). A randomized double-blind trial comparing the Carillon-Implantation to a sham-procedure was initiated (34) and presented at TCT in 2018. An early analysis of 120 patients, showed a successful device implantation in $73 / 87$ patients. $2.3 \%$ of the patients suffered procedure related death and $3.5 \%$ had myocardial infarction within 30 days. At $1 \mathrm{y}$ follow-up, the as-treated device-group had significantly lowered their regurgitant volume by $-22 \%$.

\section{Transcatheter Chordae-reconstruction}

\section{NeoChord}

The NeoChord DS1000 artificial chordae-system is a beating-heart, transapically administered Chordaereplacement-device for treatment of DMR due to elongated or ruptured chordae. Through antero-lateral mini-thoracotomy the system is introduced into the LV. By grasping the free leaflet-edge, a pledgeted NeoChord is attached to the prolapsing leaflet. Using $3 \mathrm{D}$-TEEguidance the optimal chordae-length is measured. After externalization of the Sutures, the NeoChord is secured to a epicardial pledget (35). Analyses of 30 patients in the multicentric safety and performance-trial, proved procedural success in $86.7 \%$. Durable reduction in $M R$ to $\leq 2+$ at 30 days was achieved in $33.3 \%$ of the first 15 patients and $85.7 \%$ of the last 14 patients pointing at a learning-curve that needs to be kept in mind to gain reproducible results (36). Limitations for the device-use are bileaflet-prolapse or prolapse at the commissural site. Lately presented 1y-data of 213 patients at low surgical risk enrolled in the NeoChord Independent International Registry, showed a procedural success in $96.7 \%$. At 12 month follow-up, the composed end-point of procedural success, freedom from mortality, stroke, re-intervention, recurrence of severe MR, rehospitalization and worsening of functional status defined by decrease of at least 1 grade in NYHA-class, was achieved in $84 \%$ of the treated population. Best results were seen in single-segment-posterior-leaflet-prolapse (94\%), while the treatment of multisegment posterior-prolapse or anterior/ bileaflet-prolapse showed substantially worse results (82.6\% and $63.6 \%$ respectively) (37). First randomized data comparing NeoChord to surgical MV-repair (ReChordTrial) are expected to be presented by 2020 .

Table 1 provides a summary and an overview of the 
introduced transcatheter-MV-repair devices.

\section{Transcatheter MV-replacement}

As complex transcatheter MV-repair is technically challenging and combination of repairing-techniques sometimes is impossible, a plethora of TMVR-devices have been invented in the last 10 years. For just a few of them clinical data are available. Due to the complex MV apparatus and its interaction with neighboring structures, the development of these devices took longer than in TAVR. To this day only a few hundred TMVR implantations have been done, mostly in compassionate cases. None of the devices has gained FDA-approval or CE-Mark yet.

\section{TMVR in failed surgical replacement (Valve in Valve, ViV) failed Repair (Valve in Ring, ViR) or mitral annular calcification (Valve in MAC, ViMAC)}

Using a transseptal approach to implant an inverted TAVR-prosthesis in a severely calcified mitral annulus or a preexisting mitral annular ring, in mid-2010 first case reports/-series were presented with high procedural safety and success rates.

Compared to implantations in the native valve, in $\mathrm{ViV}$ (Figure 5), ViR and ViMAC, a solid annular anchor can be used to guide the procedure and keep the prosthesis in place. Knowledge of the pre-implanted prosthesis- or ring-diameter further facilitates the choice of the proper TAVR-Size. A pooled analysis of 1,017 patients undergoing ViV- or ViR-procedures, demonstrated a 30-day mortality rate of $5.4 \%$, being significantly lower than the predicted operative mortality (38). While there is consent, that transcatheter-approaches have their rationale in redomitral-valve-interventions, it is still up for debate, if transapical or transseptal access is associated with better outcome. A systematic review of 55 studies, analyzing 183 patients, came to the conclusion that mitral ViV and ViR using transapical/transseptal access, appeared to confer equal and reasonable 30-day outcomes (39). Analyses from the TMVR multicenter registry have shown in a total of 521 patients [322 $\mathrm{ViV}, 141 \mathrm{ViR}$ (Figure 6), $58 \mathrm{ViMAC}$ ] an overall technical success-rate of $87.1 \%$, this number being significantly higher in $\mathrm{ViV}$ (94.4\%) followed by ViR (80.9\%) and ViMAC (62.1\%). LVOT-Obstruction occurred by far more often in ViMAC $(39.7 \%$ vs. $5 \%$ in $\mathrm{ViR}, 2.2 \% \mathrm{ViV}$ respectively). Overall, patients with ViR and ViMAC were associated with higher rates of adverse events and mid-term mortality than patients treated with $\mathrm{ViV}$ (40). Special care needs to be addressed to avoid too low implants in the MVannulus, since pushing the anterior leaflet towards the aorta may cause LVOT-Obstruction (41). Specialized softwares offering 3D-Visualization of ECG-gated CT-scans, have shown to be a useful tool to keep a low complication-profile.

\section{TMVR in native valve}

For patients at high-surgical risk without a rigid annular scaffold and patients with valve-disease not accessible to reconstructive-treatment, TMVR offers a last therapeutic option. Biggest challenge in these cases is the anatomical variability of the MV apparatus. Since annular calcifications cannot be used to anchor the devices, different lockingsystems had to be invented. Using either a transseptal (CardiAQ, Caisson, Sapien M3 ...) or a transapical approach (Tendyne, Tiara, Intrepid, HighLife, NaviGate, MValve, AltaValve...) the valve itself or a locking-system is anchored into the MV-apparatus and finally connected to the TMVRprosthesis. The first-in-man ever implanted TMVR was CardiAQ in 2012 (42). Most data on TVMR are available on the Tendyne-Valve-System (43).

The Tendyne-Valve-System is inserted through a transapical access. A crimped prosthesis is positioned at the MV annulus, until final deployment, the valve is fully retrievable. Once opened, the tethered valve is secured to an apical pad providing additional sealing of the insertion site (Figure 7). First in-man-implantation was performed in October 2014 (45). Thirty patients enrolled in the early feasibility trial proved TMVR with Tendyne to be an effective and safe therapy for selected patients with symptomatic native MR. Twenty-three patients had FMR, 3 predominant DMR and 4 were of mixed pathology. Mean STS-Score was $7.3 \%$. At 30 days, the device-success-rate was $93.3 \%$, leaving only one patient with $M R \geq 1+$ and all other patients with complete annular sealing and no MR after Tendyne-Implantation. Reverse Left-ventricular remodeling with decreased LVEDVi/LVESVi was significant at 30 days follow-up (46). 1-year data of the first 100 patients treated with Tendyne were presented at TVT 2019.1 year survival rate was $72 \%, 98 \%$ of the patients had MR $\leq 1+, 86.5 \%$ remained at NYHA-class $\leq \mathrm{II}(47)$.

More data on TMVR are available from the APOLLO Trial comparing TMVR with the Intrepid-System to conventional surgery. The Intrepid-system is also inserted transapically and consists of a self-expandable valve. Compared to Tendyne, the valve does not have to be 
Table 1 Overview of a selection of transcatheter-MV-repair-devices

\begin{tabular}{|c|c|c|c|c|c|c|}
\hline Technique & Device & Manufacturer & Access & Indications & Approval & Trials \\
\hline \multicolumn{7}{|c|}{ Leaflet-repair edge-to-edge } \\
\hline MitraClip & & Abbott & $\begin{array}{c}\text { Transvenous- } \\
\text { transseptal }\end{array}$ & DMR/FMR & CE \& FDA & $\begin{array}{c}\text { EVOLUT; COAPT; } \\
\text { MITRA-FR; } \\
\text { MATTERHORN; } \\
\text { RESHAPE }\end{array}$ \\
\hline PASCAL & & Edwards & $\begin{array}{c}\text { Transvenous- } \\
\text { transseptal }\end{array}$ & DMR/FMR & CE & CLASP \\
\hline \multicolumn{7}{|l|}{ Annuloplasty } \\
\hline Cardioband & & Edwards & $\begin{array}{c}\text { Transvenous- } \\
\text { transseptal }\end{array}$ & FMR & CE & ACTIVE \\
\hline Millipede & & $\begin{array}{l}\text { Boston } \\
\text { Scientific }\end{array}$ & $\begin{array}{c}\text { Transvenous- } \\
\text { transseptal }\end{array}$ & FMR & - & - \\
\hline \multicolumn{7}{|l|}{ Indirect } \\
\hline Carillon & & $\begin{array}{c}\text { Cardiac } \\
\text { Dimensions }\end{array}$ & $\begin{array}{l}\text { Transvenous- } \\
\text { jugular vein }\end{array}$ & FMR & CE & AMADEUS; TITAN \\
\hline \multicolumn{7}{|c|}{ Chordae-reconstruction } \\
\hline NeoChord & & NeoChord & Transapical & DMR & CE & RECHORD \\
\hline
\end{tabular}

$\mathrm{MV}$, mitral valve. 

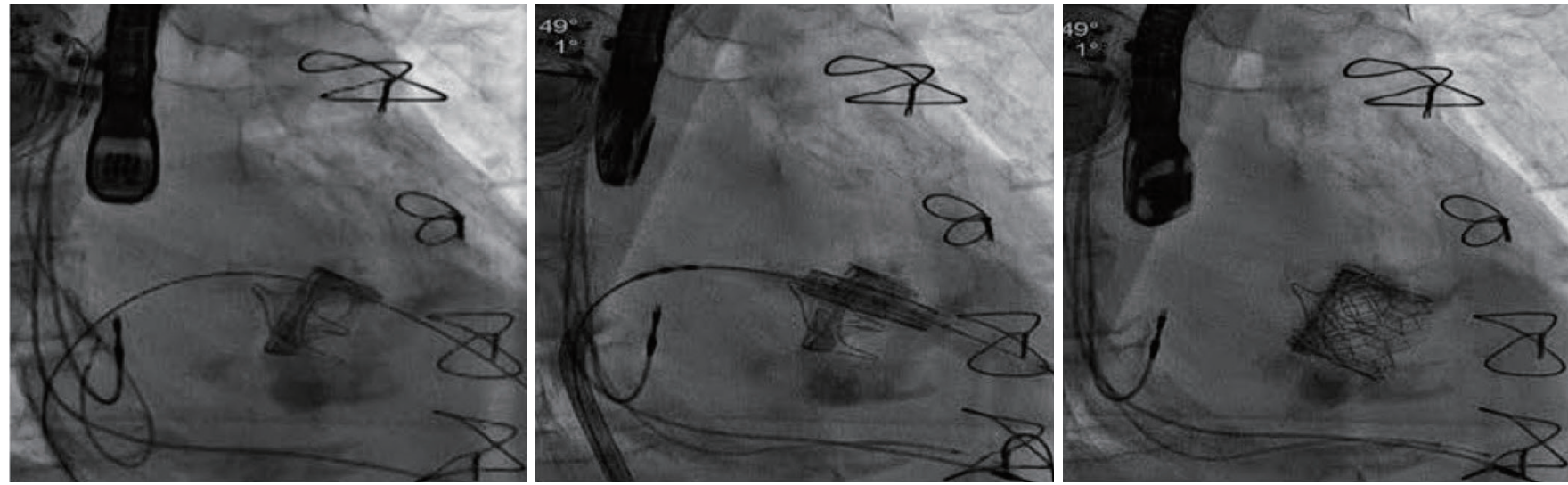

Figure $5 \mathrm{ViV}$-procedure with an inverted Edwards Sapien $326 \mathrm{~mm}$ in a degenerated Edwards Perimount $25 \mathrm{~mm}$-prosthesis in mitral position and functioning Edwards Perimount $21 \mathrm{~mm}$-prosthesis in aortic position. Special caution is required to avoid too low implantation resulting in LVOT-obstruction. LVOT, left ventricular outflow tract.

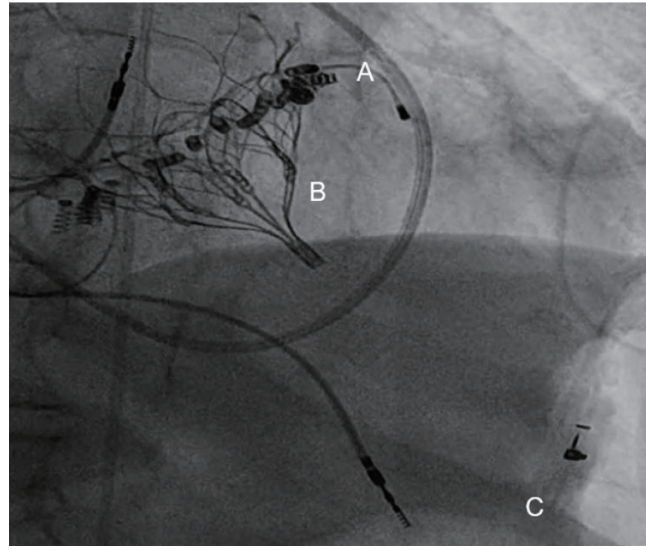

Figure 6 TMVR after transcatheter annuloplasty. (A) Cardioband; (B) Tendyne valve in ring; (C) apical tethering device. TMVR, transcatheter mitral valve replacement.

anchored at the apex, instead radial force and a barbed metal frame keep the valve in place at the MV-annulus. First in man-implantation was performed in 2014. Latest presentation of the data from the APOLLO Trial at TVT 2019 showed that out of 50 consecutively enrolled patients with an average STS-Score of $6.4 \%$, device implant was successful in 48 patients. The 30-day mortality was $14 \%$, with no disabling strokes, or repeat interventions. At latest follow-up, echocardiography confirmed mild or no residual MR in all patients who successfully received an Intrepidimplantation (48). Several feasibility and safety-trials to other TMVR-Systems are ongoing and expected to be presented in the early 2020 .

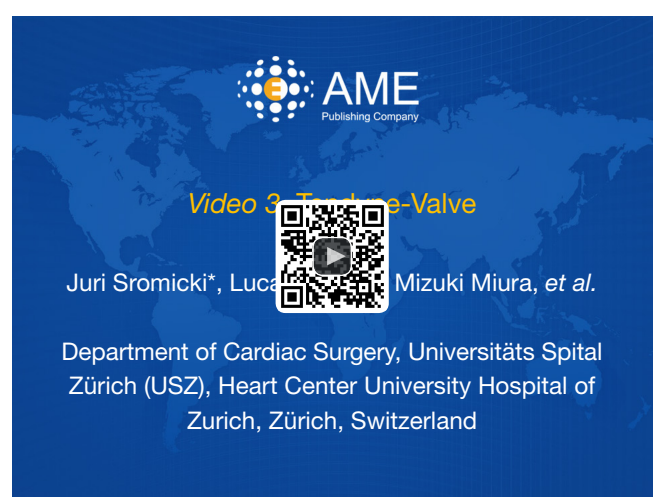

Figure 7 Tendyne-Valve (44).

Available online: http://www.asvide.com/watch/32954

Table 2 provides a summary and an overview of the introduced transcatheter-MV-replacement devices.

\section{Discussion}

Mitral regurgitation is a complex disease evolving from either malfunctioning valve-apparatus or changes in ventricular geometry resulting in annular distension. Since in open-heart-surgery MV-repair is whenever feasible the recommended first-line-treatment, early on transcatheter-devices have been invented mimicking all kinds of repair-procedures. Highlighting MitraClip, the devices have proven to be safe, effective and for certain patient-collectives not only non-inferior but superior to surgery. Ignoring the plethora of devices available on the 
Table 2 Overview of a selection of transcatheter-MV-replacement-devices

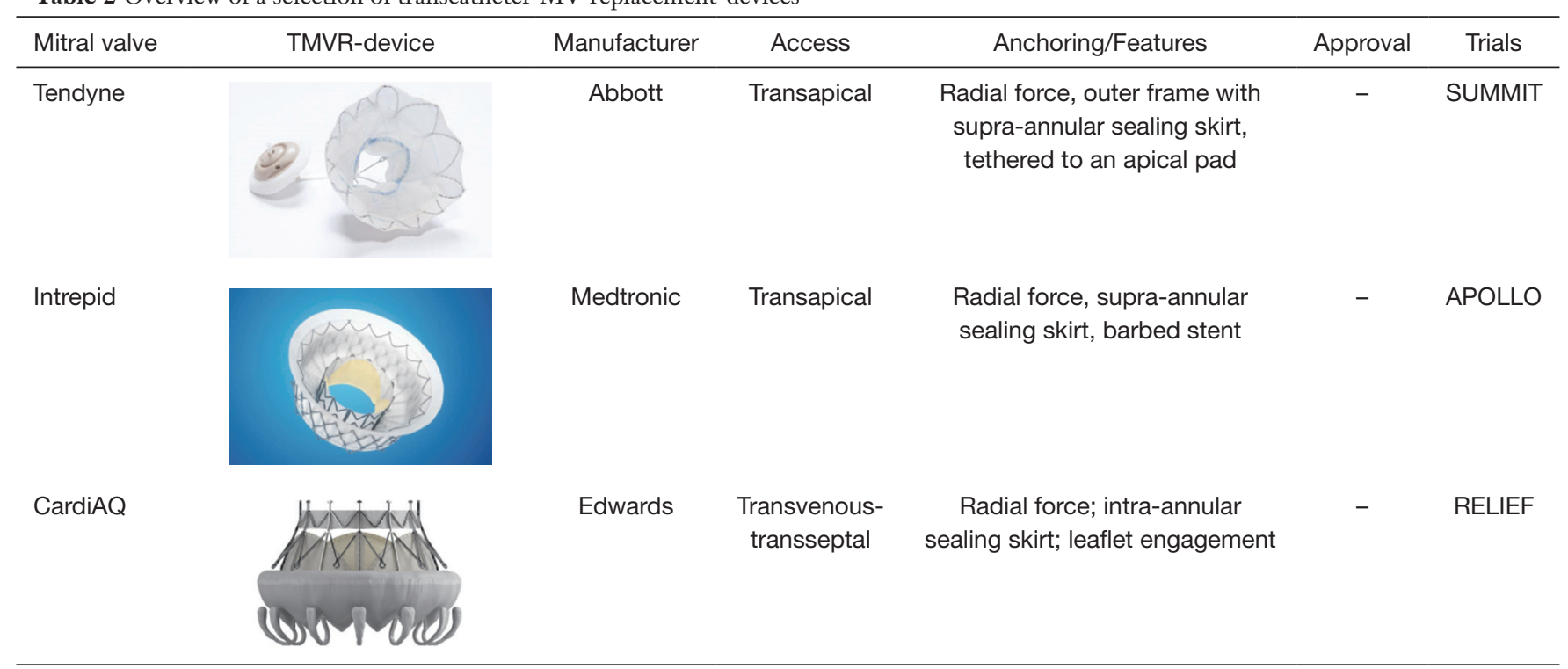

MV, Mitral valve; TMVR, transcatheter mitral valve replacement.

market, in surgery various techniques can be performed simultaneously, leading to maximal combination effect. These combinations in transcatheter-approaches are technically possible but usually rely on an experienced and skillful team of experts.

It is unlikely, that transcatheter valve-repair and TMVR will totally eliminate surgery for the treatment of MR soon, although the future in treatment of patients in heart-failure or patients with a limited life-expectancy will belong to the structural interventionalists sooner than later. A multitude of TMVR-systems are about to be tested for feasibility and safety. Once this technology is refined it is not unlikely that TMVR, other than in surgery will overcome transcatheterMV-repair, considering that treatment-options in failed transcatheter-repair-procedures are limited if surgery cannot be performed. The learning-curve to achieve as good results in transcatheter-repair as in surgery is steep. A standardized TMVR-approach that fits for the vast majority of MRpatients certainly would make structural interventions on the MV easier and accessible to more interventionalists worldwide. Results on feasibility and safety for TMVRdevices are duly expected and may significantly change future approaches for $\mathrm{MV}$-interventions.

\section{Acknowledgments}

Funding: None.

\section{Footnote}

Provenance and Peer Review: This article was commissioned by the Guest Editors (Filip Casselman and Johan van der Merwe) for the series "Aortic and Mitral Valve Innovative Surgery" published in fournal of Visualized Surgery. The article has undergone external peer review.

Conflicts of Interest: All authors have completed the ICMJE uniform disclosure form (available at http://dx.doi. org/10.21037/jovs.2019.09.05). The series "Aortic and Mitral Valve Innovative Surgery" was commissioned by the editorial office without any funding or sponsorship. MM reports and being a consultant for Japan Lifeline. MT reports personal fees from Abbott Vascular, personal fees from Boston Scientific, personal fees and other from 4tech, personal fees from Edwards Lifesciences, personal fees from Mitraltech, personal fees from CoreMedic, personal fees from Swissvortex, outside the submitted work. FM reports grants and personal fees from Abbott, grants and personal fees from Medtronic, grants, personal fees and other from Edwards Lifesciences, grants from Biotronik, grants from Boston Scientific Corporation, grants from NVT, grants from Terumo, personal fees and other from Perifect, personal fees from Xeltis, personal fees and other from Transseptal solutions, personal fees and other from Cardiovalve, personal fees and other from Magenta, other from 4Tech, other from Coregard, other from SwissVortex, 
other from Occlufit, outside the submitted work. The authors have no other conflicts of interest to declare.

Ethical Statement: The authors are accountable for all aspects of the work in ensuring that questions related to the accuracy or integrity of any part of the work are appropriately investigated and resolved.

Open Access Statement: This is an Open Access article distributed in accordance with the Creative Commons Attribution-NonCommercial-NoDerivs 4.0 International License (CC BY-NC-ND 4.0), which permits the noncommercial replication and distribution of the article with the strict proviso that no changes or edits are made and the original work is properly cited (including links to both the formal publication through the relevant DOI and the license). See: https://creativecommons.org/licenses/by-nc-nd/4.0/.

\section{References}

1. Dziadzko V, Clavel MA, Dziadzko M, et al. Outcome and undertreatment of mitral regurgitation: a community cohort study. Lancet 2018;391:960-9.

2. Goliasch G, Bartko PE, Pavo N, et al. Refining the prognostic impact of functional mitral regurgitation in chronic heart failure. Eur Heart J 2018;39:39-46.

3. Weiner MM, Hofer I, Lin HM, et al. Relationship among surgical volume, repair quality, and perioperative outcomes for repair of mitral insufficiency in a mitral valve reference center. J Thorac Cardiovasc Surg 2014;148:2021-6.

4. Carpentier A. Cardiac valve surgery--the "French correction". J Thorac Cardiovasc Surg 1983;86:323-37.

5. Baumgartner H, Falk V, Bax JJ, et al. 2017 ESC/EACTS Guidelines for the management of valvular heart disease. Kardiol Pol 2018;76:1-62.

6. Nishimura RA, Otto CM, Bonow RO, et al. 2014 AHA/ ACC Guideline for the Management of Patients With Valvular Heart Disease: a report of the American College of Cardiology/American Heart Association Task Force on Practice Guidelines. Circulation 2014;129:e521-643.

7. Alfieri O, Maisano F, De Bonis M, et al. The doubleorifice technique in mitral valve repair: a simple solution for complex problems. J Thorac Cardiovasc Surg 2001;122:674-81.

8. Lim DS, Reynolds MR, Feldman T, et al. Improved functional status and quality of life in prohibitive surgical risk patients with degenerative mitral regurgitation after transcatheter mitral valve repair. J Am Coll Cardiol 2014;64:182-92.

9. Sromicki J, Vicentini L, Miura M, et al. MitraClip. Asvide 2019;6:267. Available online: http://www.asvide.com/ watch/32952

10. Miura M, Gavazzoni M, Taramasso M, et al. Possible Transmitral Pressure Gradient Elevation in MitraClip XTR. Can J Cardiol 2019;35:544.e15-544.e17.

11. Feldman T, Wasserman HS, Herrmann HC, et al. Percutaneous mitral valve repair using the edge-to-edge technique: six-month results of the EVEREST Phase I Clinical Trial. J Am Coll Cardiol 2005;46:2134-40.

12. Feldman T, Foster E, Glower D. D, et al. Percutaneous repair or surgery for mitral regurgitation. $\mathrm{N}$ Engl J Med 2011;364:1395-406.

13. Feldman T, Kar S, Elmariah S, et al. Randomized Comparison of Percutaneous Repair and Surgery for Mitral Regurgitation: 5-Year Results of EVEREST II. J Am Coll Cardiol 2015;66:2844-54.

14. Glower DD, Kar S, Trento A, et al. Percutaneous mitral valve repair for mitral regurgitation in high-risk patients: results of the EVEREST II study. J Am Coll Cardiol 2014;64:172-81.

15. Maisano F, Franzen O, Baldus S, et al. Percutaneous mitral valve interventions in the real world: early and 1-year results from the ACCESS-EU, a prospective, multicenter, nonrandomized post-approval study of the MitraClip therapy in Europe. J Am Coll Cardiol 2013;62:1052-61.

16. Stone GW, Lindenfeld J, Abraham WT, et al. Transcatheter Mitral-Valve Repair in Patients with Heart Failure. N Engl J Med 2018;379:2307-18.

17. Obadia J. F, Messika-Zeitoun D, Leurent G, et al. Percutaneous Repair or Medical Treatment for Secondary Mitral Regurgitation. N Engl J Med 2018;379:2297-306.

18. Grayburn PA, Sannino A, Packer M. Proportionate and Disproportionate Functional Mitral Regurgitation: A New Conceptual Framework That Reconciles the Results of the MITRA-FR and COAPT Trials. JACC Cardiovasc Imaging 2019;12:353-62.

19. Praz F, Spargias K, Chrissoheris M, et al. Compassionate use of the PASCAL transcatheter mitral valve repair system for patients with severe mitral regurgitation: a multicentre, prospective, observational, first-in-man study. Lancet 2017;390:773-80.

20. Lim DS, Kar S, Spargias K, et al. Transcatheter Valve Repair for Patients With Mitral Regurgitation: 30-Day Results of the CLASP Study. JACC Cardiovasc Interv 
2019;12:1369-78.

21. Wong VM, Wenk JF, Zhang Z, et al. The effect of mitral annuloplasty shape in ischemic mitral regurgitation: a finite element simulation. Ann Thorac Surg 2012;93:776-82.

22. Taramasso M, Inderbitzin DT, Guidotti A, et al. Transcatheter direct mitral valve annuloplasty with the Cardioband system for the treatment of functional mitral regurgitation. Multimed Man Cardiothorac Surg 2016. doi: $10.1093 / \mathrm{mmcts} / \mathrm{mmw} 004$.

23. Sromicki J, Vicentini L, Miura M, et al. Cardioband. Asvide 2019;6:268. Available online: http://www.asvide. $\mathrm{com} /$ watch/32953

24. Messika-Zeitoun D, Nickenig G, Latib A, et al. Transcatheter mitral valve repair for functional mitral regurgitation using the Cardioband system: 1 year outcomes. Eur Heart J 2019;40:466-72.

25. Maisano F, Taramasso M, Guidotti A, et al. The Cardioband: strategies for optimal patient selection and optimised results. EuroIntervention 2016;12:Y61-63.

26. Latib A, Ancona M. B, Ferri L, et al. Percutaneous Direct Annuloplasty With Cardioband to Treat Recurrent Mitral Regurgitation After MitraClip Implantation. JACC Cardiovasc Interv 2016;9:e191-192.

27. Rogers JH, Boyd WD, Smith TW, et al. Transcatheter Mitral Valve Direct Annuloplasty with the Millipede IRIS Ring. Interv Cardiol Clin 2019;8:261-7.

28. Kay JH, Maselli-Campagna G, Tsuji KK. Surgical Treatment of Tricuspid Insufficiency. Ann Surg 1965;162:53-8.

29. Schueler R, Nickenig G. The Mitralign: strategies for optimal patient selection and optimised results. EuroIntervention 2016;12:Y67-69.

30. Nickenig G, Schueler R, Dager A, et al. Treatment of Chronic Functional Mitral Valve Regurgitation With a Percutaneous Annuloplasty System. J Am Coll Cardiol 2016;67:2927-36.

31. Besler C, Meduri CU, Lurz P. Transcatheter Treatment of Functional Tricuspid Regurgitation Using the Trialign Device. Interv Cardiol 2018;13:8-13.

32. Siminiak T, Wu JC, Haude M, et al. Treatment of functional mitral regurgitation by percutaneous annuloplasty: results of the TITAN Trial. Eur J Heart Fail 2012;14:931-8.

33. Lipiecki J, Siminiak T, Sievert H, et al. Coronary sinusbased percutaneous annuloplasty as treatment for functional mitral regurgitation: the TITAN II trial. Open Heart 2016;3:e00411.
34. Goldberg SL, Meredith I, Marwick T, et al. A randomized double-blind trial of an interventional device treatment of functional mitral regurgitation in patients with symptomatic congestive heart failureTrial design of the REDUCE FMR' study. Am Heart J 2017;188:167-74.

35. Colli A, Zucchetta F, Torregrossa G, et al. Transapical offpump mitral valve repair with Neochord Implantation (TOP-MINI): step-by-step guide. Ann Cardiothorac Surg 2015;4:295-7.

36. Seeburger J, Rinaldi M, Nielsen SL, et al. Off-pump transapical implantation of artificial neo-chordae to correct mitral regurgitation: the TACT Trial (Transapical Artificial Chordae Tendinae) proof of concept. J Am Coll Cardiol 2014;63:914-9.

37. Colli A, Manzan E, Aidietis A, et al. An early European experience with transapical off-pump mitral valve repair with NeoChord implantation. Eur J Cardiothorac Surg 2018;54:460-6.

38. Takagi H, Hari Y, Kawai N, et al. A meta-analysis of valve-in-valve and valve-in-ring transcatheter mitral valve implantation. J Interv Cardiol 2018;31:899-906.

39. Nazir S, Lohani S, Tachamo N, et al. Outcomes following transcatheter transseptal versus transapical mitral valve-invalve and valve-in-ring procedures. J Cardiovasc Thorac Res 2018;10:182-6.

40. Yoon SH, Whisenant BK, Bleiziffer S, et al. Outcomes of transcatheter mitral valve replacement for degenerated bioprostheses, failed annuloplasty rings, and mitral annular calcification. Eur Heart J 2019;40:441-51.

41. Yoon S. H, Bleiziffer S, Latib A, et al. Predictors of Left Ventricular Outflow Tract Obstruction After Transcatheter Mitral Valve Replacement. JACC Cardiovasc Interv 2019;12:182-93.

42. Søndergaard L, De Backer O, Franzen O. W, et al. Firstin-Human Case of Transfemoral CardiAQ Mitral Valve Implantation. Circ Cardiovasc Interv 2015;8:e002135.

43. Moat NE, Duncan A, Quarto C. Transcatheter mitral valve implantation: Tendyne. EuroIntervention 2016;12:Y75-77.

44. Sromicki J, Vicentini L, Miura M, et al. Tendyne-Valve. Asvide 2019;6:269. Available online: http://www.asvide. com/watch/32954

45. Perpetua EM, Reisman M. The Tendyne transcatheter mitral valve implantation system. EuroIntervention 2015;11 Suppl W:W78-9.

46. Muller DWM, Farivar RS, Jansz P, et al. Transcatheter Mitral Valve Replacement for Patients With Symptomatic 
Mitral Regurgitation: A Global Feasibility Trial. J Am Coll Cardiol 2017;69:381-91.

47. Sorajja P, Moat N, Badhwar V, et al. Initial Feasibility Study of a New Transcatheter Mitral Prosthesis: The First
100 Patients. J Am Coll Cardiol 2019;73:1250-60.

48. Bapat V, Rajagopal V, Meduri C, et al. Early Experience With New Transcatheter Mitral Valve Replacement. J Am Coll Cardiol 2018;71:12-21.

doi: 10.21037 /jovs.2019.09.05

Cite this article as: Sromicki J, Vicentini L, Miura M, Pozzoli A, Van Hemelrijck M, Taramasso M, Maisano F. Transcatheter approaches for mitral valve regurgitation. J Vis Surg 2019;5:78. 